\title{
METRIC PROPERTIES OF A CLASS OF QUADRATIC DIFFERENTIAL FORMS
}

\author{
P. O. BELL
}

Introduction. In the present paper a new invariant quadratic differential form $\Omega$ is geometrically defined for a general pair of surfaces $S, S^{\prime}$ whose corresponding points $x, x^{\prime}$ determine the metric normal to $S$ at $x$. The ratio of the form $\Omega$ to the first fundamental form $d s^{2}$ of $S$, in which $\Omega$ and $d s^{2}$ are defined for a common arc element of $S$ at $x$, is found to be independent of the direction of the element if and only if the surface $S^{\prime}$ is the locus of the center of mean curvature of $S$; the ratio thus determined is the Gaussian curvature $K$ of $S$ at $x$. We introduce at a point $x$ of $S$ the concept of conjugate elements of a given arc element of a conjugate net and prove that the form $\Omega$ for an arbitrary arc element is identical with the form $K d s^{2}$ for either conjugate element if and only if the surface $S^{\prime}$ is the plane net at infinity. The principal directions at $x$ of the tensor whose components are the coefficients of the form $\Omega$ are the classical principal directions of $S$ at $x$ for an arbitrary choice of $S^{\prime}$. Finally, we characterize the net of lines of mean-curvature of $S$ and the mean-conjugate net of $S$ as integral nets of equations of the form $\Omega=0$, in which the forms $\Omega$ are defined with respect to certain geometrically determined transforms $S^{\prime}$ of $S$. The method of the present paper employs dual systems of linear homogeneous equations of the first order in compact forms which facilitate the use of a tensor notation with homogeneous cartesian point and plane coordinates.

1. The fundamental differential equations. The rectangular cartesian coordinates of a generic point $x$ of an analytic surface $S$ are defined by single-valued functions of two independent parameters $u^{1}, u^{2}$,

$$
x^{i}=x^{i}\left(u^{1}, u^{2}\right), \quad i=0,1,2 .
$$

Let $g_{\alpha \beta}$ and $g^{\alpha \beta}$ denote the covariant and contravariant metric tensors of $S$, respectively, and let $d_{\alpha \beta}$ denote the second fundamental covariant tensor of $S$. It is known $[1, \mathrm{p} .220]^{1}$ that the direction cosines $z^{i}$ of the normal to $S$ at $x$ and the functions $x^{i}$ are solutions of the differential equations

$$
\frac{\partial^{2} x}{\partial u^{\beta} \partial u^{\alpha}}=\left\{\begin{array}{c}
\gamma \\
\alpha \beta
\end{array}\right\} \frac{\partial x}{\partial u^{\alpha}}+d_{\alpha \beta} z, \quad \frac{\partial z}{\partial u^{\alpha}}=\left(\begin{array}{c}
\beta \\
3 \alpha
\end{array}\right) \frac{\partial x}{\partial u^{\beta}}, \quad \alpha, \beta, \gamma=1,2,
$$

Received by the editors March 23, 1945.

${ }^{1}$ Numbers in brackets refer to the bibliography at the end of the paper. 
where the functions

$$
\left\{\begin{array}{c}
\gamma \\
\alpha \beta
\end{array}\right\}
$$

are the Christoffel symbols of the second kind formed with respect to the first fundamental form of $S$ and the functions

$$
\left(\begin{array}{c}
\beta \\
3 \alpha
\end{array}\right)
$$

are components of the mixed tensor defined by

$$
\left(\begin{array}{c}
\beta \\
3 \alpha
\end{array}\right)=-d_{\alpha \gamma} g^{\gamma \beta} \text {. }
$$

Homogeneous cartesian coordinates of a finite point of space may be obtained by adjoining to the three ordinary rectangular coordinates $x^{0}, x^{1}, x^{2}$ a fourth coordinate $x^{3}=1$. For a point at infinity the homogeneous cartesian coordinates are of the form $x^{0}, x^{1}, x^{2}, 0$. The point at infinity on the normal to $S$ at $x$ has homogeneous cartesian coordinates $z^{0}, z^{1}, z^{2}, 0$. As $x$ moves over $S$ this point at infinity describes the plane net $S_{z}$ at infinity. We observe that the system (1.1) is satisfied by each pair of homogeneous coordinates $x^{i}, z^{i}, i=0,1,2,3$. Thus the surface $S$ and the corresponding plane net $S_{z}$ at infinity generated by the infinite point on the normal to $S$ at $x$ are integral surfaces of the system (1.1).

Let us define points $x_{0}, x_{1}, x_{2}, x_{3}$ by the relations

$$
x=x_{0}, \quad \frac{\partial x_{0}}{\partial u^{\alpha}}=x_{\alpha}, \quad \alpha=1,2, \quad z=x_{3} .
$$

These relations enable us to put the system (1.1) in the form of the following system of linear homogeneous differential equations of the first order,

$$
\frac{\partial x_{i}}{\partial u^{\alpha}}-\left(\begin{array}{c}
h \\
i \alpha
\end{array}\right) x_{h}=0, \quad i, h=0,1,2,3 ; \alpha=1,2 \text {, }
$$

where

$$
\begin{gathered}
\left(\begin{array}{c}
h \\
0 \alpha
\end{array}\right)=\delta_{\alpha,}^{h},\left(\begin{array}{c}
0 \\
i \alpha
\end{array}\right)=0,\left(\begin{array}{c}
3 \\
3 \alpha
\end{array}\right)=0 ; \\
\left(\begin{array}{c}
\gamma \\
\alpha \beta
\end{array}\right)=\left\{\begin{array}{c}
\gamma \\
\alpha \beta
\end{array}\right\}, \quad\left(\begin{array}{c}
3 \\
\alpha \beta
\end{array}\right)=d_{\alpha \beta}, \quad\left(\begin{array}{c}
\beta \\
3 \alpha
\end{array}\right)=-d_{\alpha \gamma g^{\gamma \beta}, \quad \alpha, \beta, \gamma=1,2 .}
\end{gathered}
$$


Let $|x|$ denote the determinant whose elements are the functions $x_{i}^{(p)}$ and let $\xi_{i}^{\xi}$ denote the normalized cofactor of $x_{j}^{s}$ in $|x|$, defined by the relations

$$
\xi_{i}^{r} x_{h}^{r}=\delta_{h}^{r}, \quad h, r=0,1,2,3,
$$

in which the right members are the Kronecker deltas. The functions $\xi_{i}^{r}, i=0,1,2,3$, form a set of homogeneous cartesian plane coordinates of the plane determined by the three points $x_{h}, h \neq r$. Differentiating (1.4) with respect to $u^{\alpha}$ and making use of (1.3), we obtain

$$
x_{h}^{i} \frac{\partial \xi_{i}^{r}}{\partial u^{\alpha}}+\left(\begin{array}{c}
r \\
h \alpha
\end{array}\right)|x|=0 .
$$

On forming the inner product of the left member of this equation with $\xi_{k}^{n}$ and dividing by $|x|$, we find that the plane coordinates $\xi_{\mathfrak{k}}^{r}$ are solutions of the system of equations

$$
\frac{\partial \xi^{r}}{\partial u^{\alpha}}+\xi^{h}\left(\begin{array}{c}
r \\
h \alpha
\end{array}\right)=0, \quad \alpha=1,2 .
$$

A relation of the form

$$
x^{\prime}=z+k x,
$$

where $k$ is an arbitrary function of $u^{1}, u^{2}$, defines the general coordinates of a point $x^{\prime}$ which is collinear with $x$ and $z$ and generates a surface $S^{\prime}$ as $u^{1}, u^{2}$ vary. For the sake of convenience we denote the points $x, x_{1}, x_{2}, x^{\prime}$ by $y_{0}, y_{1}, y_{2}, y_{3}$, respectively, so that the fundamental differential equations (1.3) may be written in the form

$$
\frac{\partial y_{i}}{\partial u^{\alpha}}-\left(\begin{array}{c}
h \\
i \alpha
\end{array}\right)^{\prime} y_{h}=0 ; \quad i, h=0,1,2,3 ; \alpha=1,2,
$$

in which

$$
\begin{gathered}
\left(\begin{array}{c}
h \\
0 \alpha
\end{array}\right)^{\prime}=\delta_{\alpha}^{h}, \quad\left(\begin{array}{c}
0 \\
\alpha \beta
\end{array}\right)^{\prime}=-k\left(\begin{array}{c}
3 \\
\alpha \beta
\end{array}\right),\left(\begin{array}{c}
\gamma \\
\alpha \beta
\end{array}\right)^{\prime}=\left\{\begin{array}{c}
\gamma \\
\alpha \beta
\end{array}\right\}, \quad\left(\begin{array}{c}
0 \\
3 \alpha
\end{array}\right)^{\prime}=\frac{\partial k}{\partial u^{\alpha}}, \\
\left(\begin{array}{c}
\alpha \\
3 \alpha
\end{array}\right)^{\prime}=k+\left(\begin{array}{c}
\alpha \\
3 \alpha
\end{array}\right), \quad\left(\begin{array}{c}
3 \\
\alpha \beta
\end{array}\right)^{\prime}=\left(\begin{array}{c}
3 \\
\alpha \beta
\end{array}\right), \quad\left(\begin{array}{c}
3 \\
3 \alpha
\end{array}\right)^{\prime}=0, \quad \alpha, \beta, \gamma=1,2 ; \\
\left(\begin{array}{c}
\beta \\
3 \alpha
\end{array}\right)^{\prime}=\left(\begin{array}{c}
\beta \\
3 \alpha
\end{array}\right), \quad \alpha, \beta=1,2, \alpha \neq \beta .
\end{gathered}
$$

Let $|y|$ denote the determinant whose elements are the functions $y_{i}^{p}, i, p=0,1,2,3$, and let $\eta_{i}^{j}$ denote the normalized cofactor of $y_{j}^{i}$ 
in $|y|$. The functions $\eta_{\xi}^{j}, i=0,1,2,3$, are homogeneous plane coordinates of the plane determined by the three points $y_{h}, h \neq r$. The differential equations satisfied by the functions $\eta_{i}^{r}$ are easily found to be

$$
\frac{\partial \eta^{r}}{\partial u}+\eta^{h}\left(\begin{array}{c}
r \\
h \alpha
\end{array}\right)^{\prime}=0 .
$$

2. The invariant quadratic differential form. Let $x^{\prime}, X^{\prime}$ denote the points of $S^{\prime}$ whose curvilinear coordinates are $u^{1}, u^{2}$ and $u^{1}+d u^{1}$, $u^{2}+d u^{2}$, respectively, and let $\pi, p$ denote the corresponding tangent planes of $S$. Let $l$ denote the line joining the points $x^{\prime}$ and $X^{\prime}$ and let $y$ and $Y$ denote the intersections of $l$ with the planes $\pi$ and $p$, respectively. We prove the following theorem.

THEOREM 1. The principal part of the cross ratio $\left(x^{\prime}, y, X^{\prime}, Y\right)$ is the quadratic differential invariant

$$
\Omega=a_{\alpha \beta} d u^{\alpha} d w^{\beta},
$$

where $a_{\alpha \beta}$ is the tensor defined by

$$
a_{\alpha \beta}=k d_{\alpha \beta}-h_{\alpha \beta},
$$

in which $d_{\alpha \beta}$ is the second fundamental tensor of $S$ and $h_{\alpha \beta}$ is the first fundamental tensor of the spherical representation of $S$.

Except for terms of order at least two, the point coordinates of $X^{\prime}$ and the plane coordinates of $\pi$ and $p$ are given by

and

$$
X^{\prime}=y_{3}+\left(\begin{array}{c}
h \\
3 \alpha
\end{array}\right)^{\prime} y_{h} d u^{\alpha}, \quad\left(\begin{array}{c}
3 \\
3 \alpha
\end{array}\right)^{\prime}=0,
$$

$$
\pi=\eta^{3}, \quad p=\eta^{3}-\eta^{h}\left(\begin{array}{c}
3 \\
h \alpha
\end{array}\right)^{\prime} d u^{\alpha},
$$

respectively. The homogeneous cartesian coordinates of $y$, except for terms of order at least two, are obviously given by the form

$$
y=\left(\begin{array}{c}
h \\
3 \alpha
\end{array}\right)^{\prime} y_{h} d u^{\alpha} .
$$

The coordinates of $Y$ are defined by a relation of the form

$$
Y=y+\Omega y_{3}
$$

such that the condition $Y^{i} p_{i}=0$ is fulfilled. The function $\Omega$ is, therefore, the root of the equation 
1945]

$$
\left(\Omega y_{3}^{i}+\left(\begin{array}{c}
h \\
3 \beta
\end{array}\right)^{\prime} y_{h}^{i} d u^{\beta}\right)\left(\begin{array}{c}
3 \\
\eta_{i}
\end{array}-\eta_{i}^{h}\left(\begin{array}{c}
3 \\
h \alpha
\end{array}\right)^{\prime} d u^{\alpha}\right)=0 .
$$

Since $y_{j}^{q} \eta_{i}^{p}=\delta_{j}^{p}$, multiplication of the factors of the left member yields

$$
\Omega=\left(\begin{array}{c}
h \\
3 \beta
\end{array}\right)^{\prime}\left(\begin{array}{c}
3 \\
h \alpha
\end{array}\right)^{\prime} d u^{\beta} d u^{\alpha}
$$

On making use of the relations between corresponding coefficients of (1.7) and (1.3) we obtain the form

$$
\Omega=a_{\alpha \beta} d u^{\alpha} d u^{\beta},
$$

where $a_{\alpha \beta}=k d_{\alpha \beta}-g^{\delta} \gamma d_{\delta \beta} d_{\gamma \alpha}$. It is known $[1$, p. 253] that the first fundamental tensor of Gauss's spherical representation of $S$ is defined by

$$
h_{\alpha \beta}=g^{\delta} \gamma d_{\delta \beta} d_{\gamma \alpha} .
$$

The proof of the theorem is, therefore, complete.

3. New geometric characterizations of the form $K d s^{2}$. The plane at infinity is the surface $S^{\prime}$ for which $k=0$. The associated tensor $a_{\alpha \beta}$ is, therefore, defined by

$$
a_{\alpha \beta}=-h_{\alpha \beta},
$$

and the invariant $-\Omega$ is identical with the first fundamental form of the spherical representation of $S$.

It is known that for a unique conjugate parametric net, namely, the mean-conjugate net, the first fundamental form of the spherical representation is expressible in the form

$$
d \bar{s}^{2}=\sigma^{2}\left(g_{11}\left(d u^{1}\right)^{2}-2 g_{12} d u^{1} d u^{2}+g_{22}\left(d u^{2}\right)^{2}\right) .
$$

Let us determine all of the parametric nets on an unspecialized surface $S$ for which $\Omega$ is expressible in the form

$$
\Omega=\phi\left(g_{11}\left(d u^{1}\right)^{2}-2 g_{12} d u^{1} d u^{2}+g_{22}\left(d u^{2}\right)^{2}\right) .
$$

The conditions to be fulfilled are represented by the relations

$$
h_{11} / g_{11}=-h_{12} / g_{12}=h_{22} / g_{22} \text {. }
$$

It is known $[1$, p. 253$]$ that the tensor $h_{\alpha \beta}$ may be expressed in terms of the first and second fundamental tensors by means of the relation

$$
h_{\alpha \beta}=d_{\alpha \beta} K_{m}-g_{\alpha \beta} K
$$

in which $K$ and $K_{m}$ are the Gaussian curvature and mean curvature of 
$S$ defined by

$$
\begin{aligned}
K & =\left(d_{11} d_{22}-d_{12}^{2}\right) / g, \\
K_{m} & =2\left(g_{11} d_{22}-g_{12} d_{12}\right) / g .
\end{aligned}
$$

Equations (3.3), if $h_{\alpha \beta}$ is replaced by the right members of (3.4), assume the forms

$$
\begin{aligned}
& K_{m}\left(g_{22} d_{11}-g_{11} d_{22}\right)=0, \\
& K_{m}\left(d_{11} g_{12}+g_{11} d_{12}\right)=2 K g_{11} g_{12}, \\
& K_{m}\left(d_{22} g_{12}+g_{22} d_{12}\right)=2 K g_{12} g_{22} .
\end{aligned}
$$

On substituting the right members of (3.5) in equations (3.6) and simplifying, we obtain the relations

$$
\begin{aligned}
& g_{22} d_{11}-g_{11} d_{22}=0 \\
& d_{11} d_{12}=d_{12} d_{22}=0
\end{aligned}
$$

These relations are satisfied if $d_{11}=d_{22}=0, d_{12} \neq 0$, that is, if the asymptotic curves of $S$ are parametric. If, however, the asymptotic curves of $S$ are not the parametric curves of $S$, the first of equations (3.7) is the condition that the parametric net be a duametric net $[2$, p. 308]; the second and third conditions insure that the parametric net be a conjugate net. Hence, in this case, the parametric net is the unique conjugate duametric net of $S$, that is, the mean-conjugate net of $S$. We have, therefore, that $\Omega$ assumes the form (3.2) when $S^{\prime}$ is the plane net at infinity if, and only if, the parametric net of $S$ is either the asymptotic net or the mean conjugate net. For the case of the asymptotic parametric net $\phi=K$ and for the mean conjugate parametric net $\phi=-K$.

The result described above in terms of the asymptotic parametric net leads to the following geometric determination of the invariant $K d s^{2}$. Let $C_{\lambda}, C_{-\lambda}$ denote the curves which pass through $x$ of the conjugate net defined by

$$
\left(d u^{2}\right)^{2}-\lambda^{2}\left(d u^{1}\right)^{2}=0
$$

whose directions at $x$ are $\lambda,-\lambda$, respectively. Let $x$ and $P$ denote the points of $S$ whose curvilinear coordinates are $u^{1}, u^{2}$ and $u^{1}+d u^{1}$, $u^{2}+d u^{2}$, respectively. The $u^{1}$ and $u^{2}$ asymptotic curves which pass through $P$ intersect the curve $C_{-\lambda}$ in the points $Q, Q^{\prime}$ whose curvilinear coordinates are $u^{1}-d u^{1}, u^{2}+d u^{2}$, and $u^{1}+d u^{1}, u^{2}-d u^{2}$, respectively. We shall call the elements $x Q$ and $x Q^{\prime}$ the conjugate elements of $x P$. We are able now to describe our characterization of $K d s^{2}$ by means of the following theorem. 
THEOREM 2. The form $\Omega$ for an arbitrary arc element $x P$ of a conjugate net is identical with the form $K d s^{2}$ for either conjugate element $x Q$ or $x Q^{\prime}$ if and only if the surface $S^{\prime}$ is the plane net at infinity.

An analogous characterization of $-K d s^{2}$ can obviously be formulated with reference to the mean-conjugate parametric net of $S$, but we shall not describe this result here.

Another geometric characterization of $K d s^{2}$ arises from the determination of the surface $S^{\prime}$ such that the invariant $\Omega$ of $S, S^{\prime}$ is expressible in the form

$$
\Omega=\sigma d s^{2} .
$$

Just one such surface $S^{\prime}$ exists since the equations

$$
k d_{\alpha \beta}-h_{\alpha \beta}=\sigma g_{\alpha \beta}
$$

possess a unique solution $(k, \sigma)$. For, on substituting the right member of (3.4) for $h_{\alpha \beta}$ in (3.10) we find the equations

$$
\left(k-K_{m}\right) d_{\alpha \beta}=(\sigma-K) g_{\alpha \beta},
$$

which hold for an unspecialized surface $S$ if, and only if,

$$
k=K_{m}, \quad \sigma=K .
$$

Hence, we have the following theorem.

THEOREM 3. The differential form $\Omega$ of $S, S^{\prime}$ and the first fundamental form of $S$, defined with respect to a common direction, are related by the equation

$$
\Omega=\sigma d s^{2}
$$

if and only if $S^{\prime}$ is the locus of the center of mean curvature of $S$. The associated function $\sigma$ is the Gaussian curvature of $S$ at $x$.

4. The principal directions, the lines of mean curvature, and the mean conjugate net of $S$. Since we have

$$
a_{\alpha \beta}=k d_{\alpha \beta}-h_{\alpha \beta}, \quad h_{\alpha \beta}=d_{\alpha \beta} K_{m}-g_{\alpha \beta} K,
$$

we may write

$$
a_{\alpha \beta}=\left(k-K_{m}\right) d_{\alpha \beta}+K g_{\alpha \beta} .
$$

It is obvious, in view of the form of (4.1), that if $k \neq K_{m}$, the principal directions for the tensor $a_{\alpha \beta}$ are identical with the classical principal directions of $S$.

Let us assume, for the sake of convenience, that the lines of curvature are the parametric curves of $S$. The results which we present are, 
however, independent of the choice of the parametric net. The author [2, p. 309] has called a curve a line of mean curvature of $S$ if at each of its points the normal curvature $1 / \rho$ of $S$ in the direction of the curve is equal to the arithmetic mean of the principal normal curvatures of $S$ at the point, that is, if

$$
\frac{1}{\rho}=\frac{1}{2}\left(\frac{1}{\rho_{1}}+\frac{1}{\rho_{2}}\right) \text {. }
$$

From equations (6.5) and (6.6) of $\left[2\right.$, p. 310] we find that if $g_{12}=d_{12}$ $=0$, the lines of mean curvature of $S$ are the integral curves of

$$
\Delta\left(g_{11}\left(d u^{1}\right)^{2}-g_{22}\left(d u^{2}\right)^{2}\right)=0
$$

in which $\Delta=g_{11} d_{22}-g_{22} d_{11}$. The significance of the presence of $\Delta$ in equation (4.2) is that the lines of mean curvature form a determinate net, except when $\Delta=g_{12}=d_{12}=0$; in this exceptional case $S$ is a sphere.

We inquire if there exists a surface $S^{\prime}$ for which $\Omega$ is expressible in the form

$$
\Omega=\sigma \Delta\left(g_{11}\left(d u^{1}\right)^{2}-g_{22}\left(d u^{2}\right)^{2}\right) .
$$

The affirmative answer is readily reached, for we find that the equations

$$
\begin{aligned}
& k d_{11}-h_{11}=\sigma \Delta g_{11}, \\
& k d_{12}-h_{12}=0, \\
& k d_{22}-h_{22}=-\sigma \Delta g_{22}
\end{aligned}
$$

can be satisfied simultaneously. The second equation of (4.4) is satisfied identically in $k, \sigma$ since $g_{12}=d_{12}=h_{12}=0$. The other two equations may be written in the forms

$$
\begin{aligned}
& k d_{11}-g_{22} d_{11}^{2} / g=\sigma \Delta g_{11}, \\
& k d_{22}-g_{11} d_{22}^{2} / g=-\sigma \Delta g_{22} .
\end{aligned}
$$

Solving, we find

$$
\begin{aligned}
& k=\left(d_{11 g_{22}^{2}}^{2}+g_{11}^{2} d_{22}^{2}\right) / g\left(g_{22} d_{11}+g_{11} d_{22}\right), \\
& \sigma=d_{11} d_{22} / g\left(g_{22} d_{11}+g_{11} d_{22}\right) .
\end{aligned}
$$

Making use of the expressions

$$
\begin{aligned}
K & =d_{11} d_{22} / g, \\
K_{m} & =\left(g_{11} d_{22}+g_{22} d_{11}\right) / g
\end{aligned}
$$


for the Gaussian and mean curvatures of $S$, we express $k, \sigma$ in terms of $K$ and $K_{m}$ as follows:

$$
k=K_{m}-2 K / K_{m}, \quad \sigma=K / g K_{m} .
$$

Hence we have the following theorem.

THEOREM 4. If and only if $S^{\prime}$ is characterized in relation to $S$ by (1.7) with $k$ expressed in terms of the Gaussian and mean curvatures of $S$ by the relation

$$
k=K_{m}-2 K / K_{m},
$$

the lines of mean curvature of $S$ are the integral curves of the associated differential equation $\Omega=0$.

Let us recall that a curve of the mean conjugate net is characterized by the property that at each of its points the radius of normal curvature of $S$ in the direction of the curve is the arithmetic mean of the principal radii of normal curvature of $S$ at the point, that is,

$$
\rho=\left(\rho_{1}+\rho_{2}\right) / 2 \text {. }
$$

Retaining the lines of curvature as parametric curves, the mean conjugate net is the integral net of the equation

$$
\Delta\left(d_{11}\left(d u^{1}\right)^{2}-d_{22}\left(d u^{2}\right)^{2}\right)=0 .
$$

It is not difficult to prove, by the method we have employed above, that a surface $S^{\prime}$ exists such that the associated form $\Omega$ is expressible by the relation

$$
\Omega=\sigma \Delta\left(d_{11}\left(d u^{1}\right)^{2}-d_{22}\left(d u^{2}\right)^{2}\right) .
$$

In fact, we find that

$$
k=K_{m} / 2, \quad \sigma=1 / 2 g .
$$

We are now in a position to state our concluding result.

THEOREM 5. If and only if $k$ is the arithmetic mean of the principal normal curvatures of $S$ at $x$, the integral net of the associated differential equation $\Omega=0$ is the mean conjugate net of $S$.

\section{BIBLIOGRAPHY}

1. L. P. Eisenhart, An introduction to differential geometry with use of the tensor calculus, Princeton University Press, Princeton, 1940.

2. P. O. Bell and W. C. Foreman, Euclidean applications of the projective differential geometry of the $R_{\lambda}$-correspondent, Ann. of Math. vol. 44 (1943) pp. 298-314.

The UNIVERSITY OF KANSAS 